SOURCES

Quotations from individuals identified in the text, unless otherwise noted, appear without formal citation. A list of interviews along with their dates can be found in Sources at the end of this book. Occasionally I have withheld a person's identity, either by request or because of the sensitive nature of the material.

\title{
PITCH NOMENCLATURE
}

For the pitch nomenclature used in this book, italic letters are used exclusively when discussing specific pitches within a defined range or octave. Nonitalic capital letters denote pitch classes, not specific pitches. All pitches from middle $\mathrm{C}$ and above are indicated in lowercase italics. For pitches beginning at the $\mathrm{C}$ an octave above middle $\mathrm{C}$, a superscript number indicates the number of octaves above middle $\mathrm{C}$ (i.e., $c^{2}$ indicates the $\mathrm{C}$ two octaves above middle $\mathrm{C}$ ). All pitches below middle $\mathrm{C}$ are in uppercase italics with a subscript number indicating the number of octaves below middle $\mathrm{C}$ (i.e., $C_{2}$ is the $\mathrm{C}$ two octaves below middle $\mathrm{C}$ ). 
This page intentionally left blank 